\title{
Clinical care given to victims of sexual assault at Kadoma General Hospital, Zimbabwe: a secondary data analysis, 2016
}

\author{
Stanley Tapesana ${ }^{1}$, Daniel Chirundu², Gerald Shambira', Notion Tafara Gombe ${ }^{1}$, Tsitsi Patience Juru* \\ and Tshimanga Mufuta'
}

\begin{abstract}
Background: Despite the guidelines for managing sexual assault being in place, victims of sexual assault attended to at Kadoma General Hospital consistently raised complaints related to the quality of care offered. Medicolegal data for sexual assault has been collected at the hospital since 2012. However, no analysis had been done regardless of complaints having been raised. We analysed the dataset to determine the quality of clinical care offered to sexual assault victims.

Methods: A retrospective cross-sectional study based on secondary data was conducted. Epi. Info 7 software was used to analyse data and generate frequencies, measures of central tendency and proportions.

Results: We analysed 474 medical affidavits completed between January 2014 and July 2016. Thirty percent of the victims sought care within $72 \mathrm{~h}$ of the sexual assault. Baseline HIV testing was done in 23 (22\%) and follow-up HIV test done in 2 (2\%) of the victims. Post Exposure Prophylaxis for HIV was administered to 18 (51\%), emergency contraception 9 (69\%) and forensic evidence gathered in six (5\%) of victims presenting within the prescribed $72 \mathrm{~h}$ of the sexual assault. Prophylactic antibiotics were given to 156 (33\%). There were no documented counselling sessions for all victims whilst follow up care was given to 47 (10\%) victims.

Conclusion: Suboptimal clinical care was given to victims of sexual assault during the period 2014-2016. These findings suggest possible delayed presentation by victims of sexual assault as well as suboptimal administration of prophylaxis by health care workers. We recommend adherence to guidelines in managing sexual assault. Further research to determine factors for delayed presentation among sexual assault victims and quality of care provided to them is recommended.
\end{abstract}

Keywords: Sexual Assault, Secondary Analysis, Clinical Care, Kadoma

\section{Background}

Gender-based violence (GBV) is a growing global public health concern of the twenty-first century. GBV cuts across all classes, races, ages, religions and national boundaries and contributes to social, legal and health challenges mainly affecting women [1-3]. Sexual assault is recorded as one of the worst forms of gender-based violence with long-term implications. The United $\mathrm{Na}$ tions statistical report indicates that; globally more than

\footnotetext{
*Correspondence: tsitsijuru@gmail.com

1 Department of Community Medicine, University of Zimbabwe, Harare, Zimbabwe

Full list of author information is available at the end of the article
}

250,000 cases of sexual assault recorded annually. The prevalence of sexual assault ranges from $16.3 \%$ in East Asia to $65.64 \%$ in Central sub-Saharan Africa [4]. The estimated lifetime prevalence of sexual assault among primary students between 13 and 15 years in SubSaharan Africa ranges from $9 \%$ to $33 \%$.

A variety of consequences of sexual assault on a survivor's physical and mental health have been documented. These include physical injury, sexually transmitted infections (STIs) including HIV, unwanted pregnancy, unsafe abortion, anxiety, shame, posttraumatic stress, and depression [5]. In this regard, timely provision of health care to victims of sexual assault is crucial as several 
important aspects of the care are time dependent. The World Health Organisation guidelines for management of sexual assault covers the important aspects of management that include, provision of emergency contraception, post-exposure prophylaxis for HIV and/or collection of forensic evidence [6].

In Zimbabwe, management of sexual assault is guided by the Southern African Development Community Protocol on Gender and Development as well as the National Guidelines on Management of Sexual Assault [7, 8]. According to Zimbabwe Demographic and Health Survey (ZDHS 2015), 37\% of those who experienced physical or sexual violence sought help [9].

Despite several complaints pertaining to the quality of care offered to victims having been raised, the medical affidavit dataset had never been analysed to determine the quality of clinical care offered to victims of sexual assault. We analysed the dataset in order to determine the adequacy of clinical care offered to sexual assault victims in line with the guidelines.

\section{Methods}

Study design and description of the study site

We conducted a retrospective cross-sectional study based on the secondary dataset at Kadoma General Hospital. Kadoma General Hospital is the only referral hospital therefore, all the sexual assault cases in Sanyati district are referred to this hospital.

\section{Study subjects and sample size}

The study population was made up of all the medical affidavits completed at Kadoma General Hospital from January 2014 to July 2016. The study unit was a victim's medical affidavit completed and kept at Kadoma General Hospital. Although the sample size was 357 medical affidavits we included all the 474 medical affidavits for analysis.

The variables that we investigated in our study included:

- Socio-demographics- age, sex, marital status, relationship to perpetrator, previous sexual experience and secondary sexual development characteristics

- Time to presentation- categorised as 0-24 h, 1-3 days, 4-7 days, 8-30 days, 1-3 months, > 3 months and unknown duration

- Clinical evidence of sexual penetrationcategorised as definite, very likely, probable, inconclusive but possible and no visible evidence

- Associated signs and symptoms- behaviour change, genital discharge, genital pain, blood loss, dysuria, lower abdominal pain, difficulty in walking, genital sores, pain on defaecation, genital warts and other
- Clinical management- proportion of victims who received HIV Post-exposure prophylaxis, emergency contraception, prophylactic antibiotics, forensic tests, counselling and follow-up care

- Laboratory tests done for victims- baseline HIV test, follow up HIV test, vaginal swab microscopy, pregnancy test and syphilis test

\section{Data collection and analysis}

Epi Info Companion (CDC, 2012) was used to capture data. The analysis was done using the same software. The software was used to generate frequencies, measures of central tendency and proportions. A sub analysis was done to assess the clinical care that was offered to the victims of sexual assault depending on their stage of development, prior history of sexual penetration, clinical presentation and clinical evidence of sexual assault.

\section{Ethical considerations}

The names of health care workers, victims of sexual assault and police officers were kept confidential. This was achieved by assigning an anonymous identification number to each medical affidavit of sexual assault during data capture, and analysis. We obtained permission to proceed from the Provincial Medical Director Mashonaland West Province, Kadoma District Medical Officer and the Health Studies Office.

\section{Results}

A total of 474 medical affidavits were analysed. The overall completeness of the dataset was $73 \%$.

\section{Demographic characteristics}

The median age of the victims was 14 years $\left(\mathrm{Q}_{1}=11\right.$; $\left.\mathrm{Q}_{3}=16\right)$, whilst that of perpetrators was 21 years $\left(\mathrm{Q}_{1}=18\right.$; $\mathrm{Q}_{3}=27$ ). Four hundred and seventy-three $(98.8 \%)$ of the victims were female. Seventy-four percent (204) victims of sexual assault demonstrated their relationship with perpetrators. Three hundred and thirty-one (72.6\%) of the victims were younger than 16 years while $88(19.3 \%)$ were single women and $21(4.6 \%)$ married women. Male perpetrators were 204 (99.8\%) as illustrated in Table 1 . The relationship of perpetrator to the victim was that of a boyfriend in 81 (40\%), stranger $51(25 \%)$ and uncles in $18(5 \%)$ as shown in Fig. 1.

Time to presentation and clinical evidence of penetration Time to presentation among victims of sexual assault is shown in Fig. 2. Thirty percent of the victims presented to the health facility within $72 \mathrm{~h}$ of being sexually assaulted. Twenty-four percent presented within four to seven days of being sexually assaulted whilst 154 (36\%) presented after seven days and in $43(10 \%)$ the time of the sexual assault was not known. Definite clinical evidence of 
Table 1 Demographic Characteristics of Sexual Assault Victims Kadoma General Hospital $2016(n=474)$

\begin{tabular}{lll}
\hline Variable & & $\mathrm{n}(\%)$ \\
\hline Sex of Victim & Male & $1(0.2)$ \\
Relationship of victim to perpetrator & Related & $473(99.8 \%)$ \\
& Not related & $150(74 \%)$ \\
Marital status & Child under 16 years & $331(26 \%)$ \\
& Single & $88(19.3 \%)$ \\
& Married & $21(4.61 \%)$ \\
& Divorced & $13(2.85 \%)$ \\
& Other & $2(0.44 \%)$ \\
Secondary sexual development & Widowed & $1(0.22 \%)$ \\
& Pubertal & $229(51.81 \%)$ \\
& Pre-pubertal & $125(28.28 \%)$ \\
& Mature & $86(19.46 \%)$ \\
& Post-menopausal & $2(0.45 \%)$ \\
\hline
\end{tabular}

penetration was found in 197 (44\%), very likely 99 (22\%), probable $54(12 \%)$, inconclusive but possible in $22(5 \%)$ and no visible evidence in $76(17 \%)$ of victims as illustrated in Fig. 3. Condom use by the perpetrator of sexual violence was documented in 9 (2\%) of sexual assaults.

\section{Clinical signs and symptoms in victims}

External body injury was recorded in 185 (39\%), anal injury 128 (27\%), external female genitalia injury 182 (42\%) and internal vaginal injury in 182 (42\%). Among other clinical findings were behaviour change in 25 (31\%), genital discharge 15 (19\%), blood loss 13 (16\%), dysuria $12(15 \%)$ and lower abdominal pain 12 (15\%). Genital sores and pain on defecation were documented in $4(5 \%)$ victims as shown in Fig. 4.

\section{Clinical management in sexual abuse victims}

Baseline HIV testing was done in 23 (22\%) and second HIV test done in 2 (2\%) of the victims. Ninety-eight (94\%) victims were HIV negative on baseline HIV testing while six (6\%) were HIV positive. Three victims out of eight who were initially HIV negative were found to be HIV positive on follow-up testing. Post-exposure prophylaxis for HIV was given to 18 (51\%) and emergency contraception was given to 9 (69\%) of the sexual assault victims presenting within $72 \mathrm{~h}$ of the sexual assault. Vaginal swab microscopy was done in one of the five victims who presented with a discharge. Prophylactic antibiotics were administered in 156 (33\%) of the victims. Six out of 474 (1\%) of the victims had forensic tests done. Of the 128 victims presenting within $72 \mathrm{~h}$ of the sexual assault, six (5\%) had forensic tests done. Victims of sexual assault who had follow-up care were 47 (10\%). Of all the victims attended at this institution there was no documentation of counselling sessions.

\section{Discussion}

This was a cross-sectional study based on secondary data analysis. We sought to determine the clinical care given to victims of sexual abuse at Kadoma General Hospital, Zimbabwe between 2014 and 2016. The median age of victims in our study was 14 years. Sexual assault has been reported to be more common in this age group owing to the phase of sexual development that is adolescence [10]. This is also supported by the fact that the majority (40\%) of the perpetrators in our study were boyfriends of the victims. However, our findings could be attributed to less reporting by older and married females owing to the fear of stigma and perceived social consequences associated with reporting sexual assault. In our study, females constituted $98.8 \%$ of the total victims of sexual assault. Our finding is consistent with that reported by Ige et al. [11] and Abdulkadir et al. [12] who

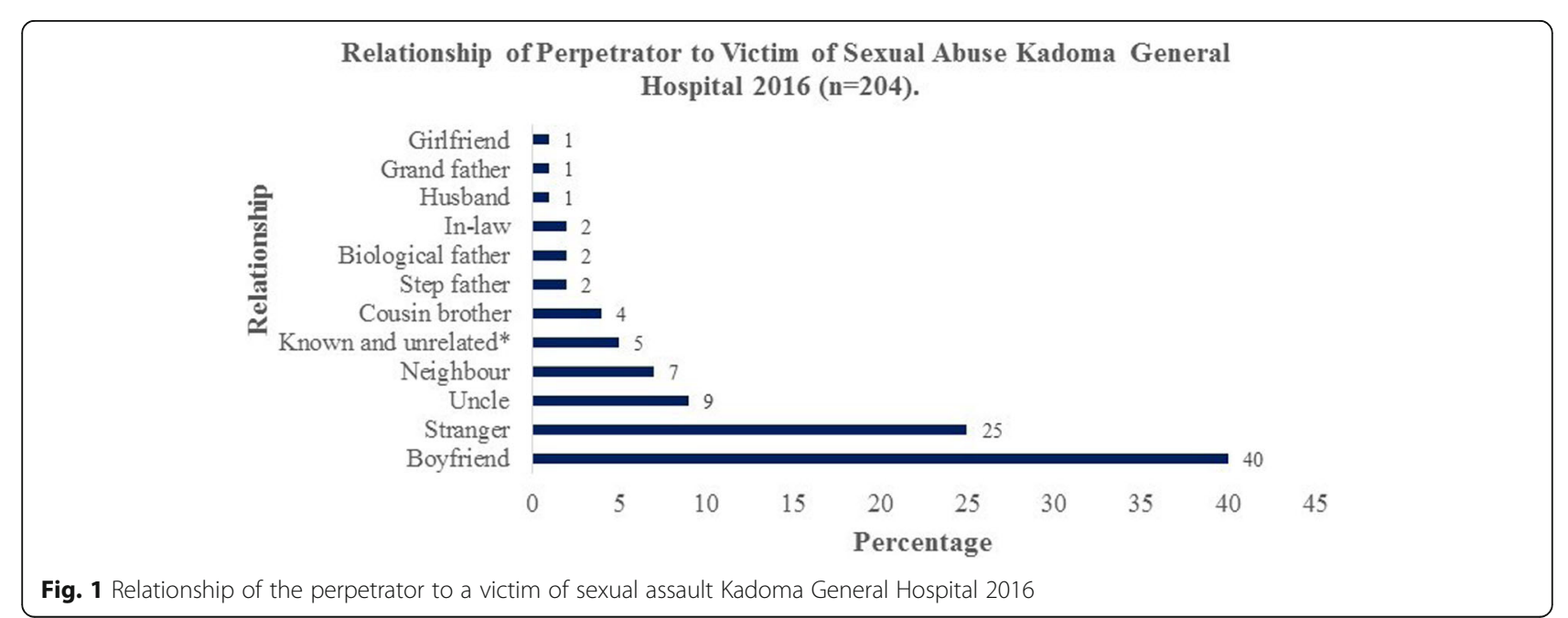


Time to Presentation to Health Facility by Victims of Sexual Abuse

Kadoma General Hospital $2016(n=429)$

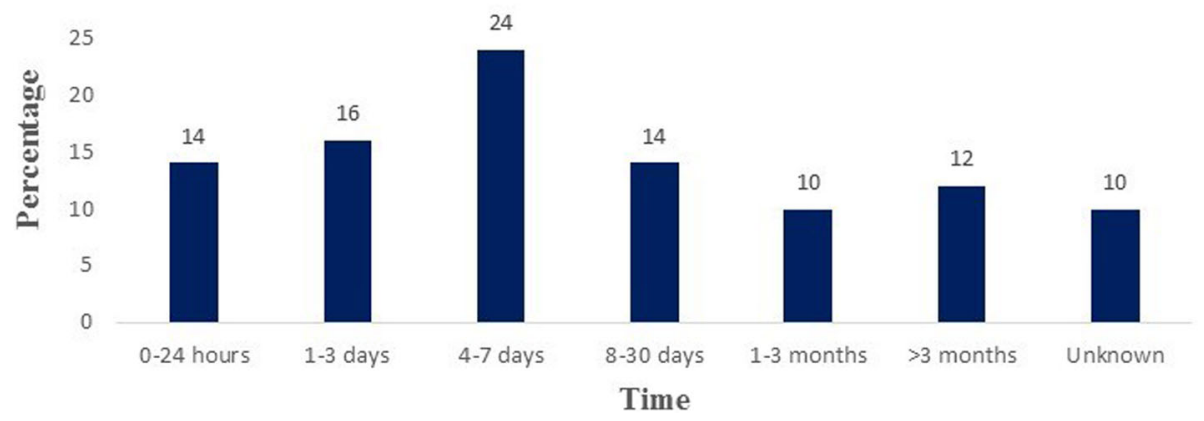

Fig. 2 Time to presentation to the health facility by victims post sexual assault Kadoma General Hospital 2016

reported a vulnerability of females to sexual assault owing to the patriarchal social norms that expose women to social injustice and exploitation. In our study, $74 \%$ of the perpetrators were known to their victims. We also found that the majority of perpetrators were boyfriends 81 (40\%), relatives 46 (21\%), stranger 51 (25\%) and neighbour 15 (7\%). Most of the victims fell prey to the people they knew unexpectedly, and this may be a result of the trust and relationship between the perpetrator that makes the victim assaulted unexpectedly. Girgira et al. [13] and Birdthistle et al. [14] reported similar findings in their studies in Ethiopia and Zimbabwe respectively.

In our study, the modal time to present to a health facility following sexual assault was between four and seven days. In Ethiopia, the median length of time taken to present to a health facility was found to be four days [13]. The delay in presentation to health facilities following sexual assault compromises medical interventions, as we expect victims to receive prophylactic therapy within $72 \mathrm{~h}$ of sexual assault to optimise the benefits. We found condom usage by perpetrators of sexual violence in $2 \%$ of the victims. The low condom usage results in a higher risk of contracting sexually transmitted infections and unwanted pregnancies among victims hence the need for optimum preventive interventions following sexual assault.
In a sub-analysis of 81 medical affidavits, genital discharge was recorded in 15 (19\%) and bleeding in 13 (16\%) of the victims. External body injury was reported in 185 (39\%), anal injury 128 (27\%), external female genital injury $182(42 \%)$ and internal vaginal injury in $182(42 \%)$. The presence of genital discharge and genital injury in these victims is substantial evidence of penetrative sexual assault. The presence of bleeding results in a breach in the mucosal surface of the genital tract hence predisposing the victims to acquire sexually transmitted infections. The importance of standard quality of care in victims of sexual assault cannot be underestimated. However, there could be more asymptomatic victims who later suffer sexual assault related complications such as subfertility and posttraumatic stress disorders. Vaginal lacerations with bleeding, hymeneal tear, and soft tissue injury have been reported in similar studies $[11,13]$.

In our study, prophylactic antibiotics were given in 36 (33\%). However, one of the victims who had a vaginal discharge was not given antibiotics. Similar studies have also reported administration of post-exposure prophylaxis for sexually transmitted infections in $53.9 \%$ of the victims [13]. Ideally, prophylactic antibiotics should be given to all victims who present with or without symptoms of sexually transmitted infections as long as there is possible evidence of penetration. This prophylaxis is

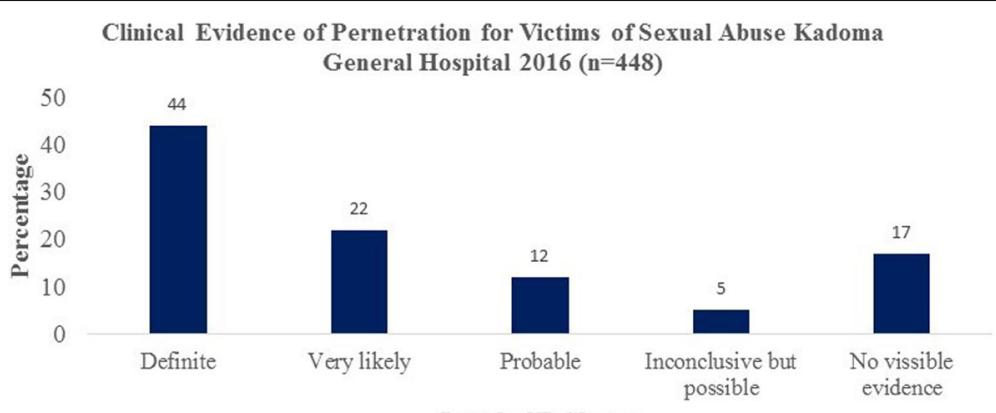

Level of Evidence

Fig. 3 Clinical evidence of penetration for victims of sexual assault Kadoma General Hospital 2016 
Clinical Signs and Symptoms in Victims of Sexual Abuse Kadoma General Hospital $2016(n=81)$

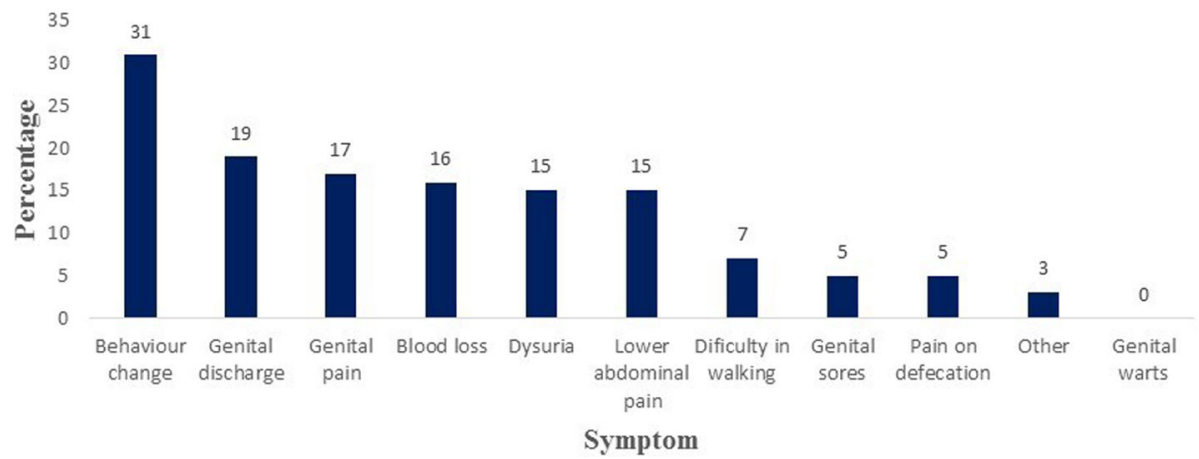

Fig. 4 Signs and symptoms among victims of sexual assault Kadoma General Hospital 2016

necessary to prevent the development of sexually transmitted infections and future sequelae associated with sexual assault [10].

The proportion of victims (30\%) who were eligible to receive Post Exposure Prophylaxis (PEP) for HIV was low. This finding was due to delayed presentation for health care by victims following sexual assault. However, among those who presented on time, only 35 (51\%) received PEP for HIV and 9 (69\%) got emergency contraception. Owing to the services being provided in a busy outpatient department there is a possibility of missing some of the essential components of the clinical care delivered to victims of sexual assault. The victims also present to the hospital with a police officer whose priority is to have the medical affidavit signed for court proceedings and once the form is completed the victim is likely to leave the hospital without receiving the full package of care as required. There is need to prioritise the acute clinical care given to victims and this can be done by a multisector approach. Birdthistle et al. [14] in a study in Zimbabwe reported a high PEP coverage (92\%) for victims presenting within $72 \mathrm{~h}$ [11]. The high PEP coverage found by Birdthistle owed to the model of service delivery at the health facility, in this case, it was a specialised clinic for sexual assault victims as compared to the outpatient model used at Kadoma General Hospital.

In our study, the proportion of victims who had baseline HIV testing was $22 \%$ which is low as we expect all victims to have a baseline HIV test. At baseline HIV testing, six (6\%) of the victims were HIV-positive. Among these victims, three were adults who were sexually active but had never been screened for HIV infection before. Owing to the high HIV prevalence in Zimbabwe there is need to screen all victims for HIV as recommended by the guidelines. Of the eight victims tested for HIV at three months' follow-ups, three became HIV-positive (38\%). Missing PEP and appropriate care in the victims could have caused transmission of
HIV in these three victims following the sexual assault. However, in settings with low HIV prevalence, the baseline and second HIV tests have been found to be negative in all victims $[9,13]$.

Our findings show that few victims were offered vaginal swab microscopy despite the overwhelming evidence of penetration and infection. Vaginal swab microscopy is essential for guiding antibiotic treatment in the victims with vaginal discharge. Despite 45 victims with definite evidence of penetration having presented within $72 \mathrm{~h}$, forensic evidence was gathered in only six $(13 \%)$ of the victims. Insufficiency of evidence during prosecution and lenient penalties for the perpetrators have resulted from the lack of adequate and convincing forensic evidence. Few of the victims (10\%) in our study received follow-up care whilst there were no proper counselling sessions documented. This finding could be attributed to the non-existence of a proper follow-up mechanism for sexual assault clients. Counselling and follow up in sexual abuse victims has been shown to be suboptimal in several studies [11, 13]. Abdulkadir et al. [12] also reported non-documentation of the management of sexually transmitted infections, emergency contraception, HIV infection and scheduled follow up in a retrospective study of 81 sexual abuse victims in Niger State.

The major limitation encountered in our study was the incompleteness of variables relating to laboratory investigations and forensic tests and this could be attributed to the poor recording practices among clinicians. The insufficiency of the dataset could also affect generalisation of our study findings.

\section{Conclusions}

We conclude that suboptimal clinical care was given to victims of sexual abuse during the period 2014-2016. These findings suggest possible delayed presentation by victims of sexual abuse as well as suboptimal administration of prophylaxis by health care workers. We 
recommend adherence to guidelines in managing sexual abuse victims. Further research to determine factors for delayed presentation for care among sexual abuse victims and quality of care provided to them is recommended.

\section{Abbreviations}

GBV: Gender-Based Violence; HIV: Human Immunodeficiency Virus; PEP: Post Exposure Prophylaxis; STI: Sexually Transmitted Infections; ZDHS: Zimbabwe Demographic and Health Survey

\section{Acknowledgements}

We would like to acknowledge the following organisations and individuals for making our study a success: Kadoma City, Kadoma General Hospital, Obert Chingozho and Blessing Banda.

\section{Funding}

Funding was sought from CDC, Atlanta Georgia.

\section{Availability of data and materials}

The data that support the findings of this study are available from Ministry of Health and Child Care (Zimbabwe) but restrictions apply to the availability of these data. Data are however available from the authors upon reasonable requests and with permission of Ministry of Health and Child Care (Zimbabwe).

\section{Authors' contributions}

ST, DC GS.TJ, NG, MT: conception, design, acquisition. ST, DC, GS: data collection, analysis and interpretation of data. ST, DC, GS wrote the first draft of the manuscript. GS, NG, TJ, MT critically revised the draft for important intellectual content. All authors read and approved the final manuscript.

\section{Authors' information}

Not applicable.

\section{Ethics approval and consent to participate}

Permission to proceed with the study was obtained from the Institutional Review Board Kadoma City and the University of Zimbabwe, Department of Community Medicine.

\section{Consent for publication}

Not applicable.

\section{Competing interests}

The authors declare no competing interests.

\section{Publisher's Note}

Springer Nature remains neutral with regard to jurisdictional claims in published maps and institutional affiliations.

\section{Author details}

${ }^{1}$ Department of Community Medicine, University of Zimbabwe, Harare, Zimbabwe. ${ }^{2}$ Kadoma City Health Department, Kadoma City, Zimbabwe.

Received: 20 February 2017 Accepted: 22 August 2017

Published online: 31 August 2017

\section{References}

1. Sanjel S. Gender-based violence: A crucial challenge for public health. Kathmandu University Med J. 2013;42(2):179-84.

2. Ellsberg $\mathrm{M}$, Jansen $\mathrm{HA}$, Heise $\mathrm{L}$, Watts $\mathrm{CH}$, Garcia-Moreno $\mathrm{C}$. Intimate partner violence and women's physical and mental health in the WHO multicountry study on women's health and domestic violence: An observational study. Lancet. 2008;371:1165-72.

3. Marsh M, Purdin S, Navani $\mathrm{S}$. Addressing sexual violence in humanitarian emergencies. Glob Public Health. 2006;1 (2):133-46.

4. Devries KM, Mak JYT, Garcia-Moreno C. The global prevalence of intimate partner violence against women. Science. 2013;340(6140):1527-8.

5. Girgira T, et al. Time to presentation, pattern and immediate health effects of alleged child sexual abuse at two tertiary hospitals in Addis Ababa, Ethiopia. BMC Public Health. 2014;14:92.
6. Kim JC, Askew I, Muvhango L, Dwane N, Abramsky T, Jan S, et al. Comprehensive care and HIV prophylaxis after sexual assault in rural South Africa: The Refentse intervention study. BMJ. 2009 March;13:2009.

7. SADC Protocol on Gender and Development. http://www.sadc.int/files/ 8713/5292/8364/Protocol_on_Gender_and_Development_2008.pdf. Accessed 13 Feb 2017

8. Protocol on the Multi-Sectoral Management of Sexual Abuse and Violence in Zimbabwe 2012. http://www.togetherforgirls.org/wp-content/uploads/ Multi_Sectoral_Protocol_2012.pdf. Accessed 2 Nov 2016.

9. Zimbabwe Demographic and Health Survey 2015. Key Indicators Zimbabwe National Statistics Agency Harare, Zimbabwe. Rockville: The DHS Program ICF International; 2016.

10. Pitche P. Child sexual abuse and sexually transmitted infections in subSaharan Africa. Med Trop (Mars). 2005;65:570e4.

11. Ige OK, Fawole Ol. Evaluating the medical care of child sexual abuse victims in a general hospital in Ibadan. Nigeria: Ghana medical journal; 2012.

12. Abdulkadir I, Umar LW, Musa HH, Musa S, Oyeniyi OA, Ayoola-Williams OM, et al. Child sexual abuse: A review of cases seen at General Hospital Suleja, Niger State. Ann Nigerian Med. 2011;5:15-9.

13. Girgira T, Tilahun B, Bacha T. Time to presentation, pattern and immediate health effects of alleged child sexual abuse at two tertiary hospitals in Addis Ababa. Ethiopia BMC Public Health. 2014;14:92.

14. Birdthistle IJ, Floyd S, Mwanasa S, Nyagadza A, Gwiza E, Glynn JR. Child sexual abuse and links to HIV and orphanhood in urban Zimbabwe 2010. J Epidemiol Community Health. 2011;65:1075e1082. doi:10.1136/jech.2009. 094359
Submit your next manuscript to BioMed Central and we will help you at every step:

- We accept pre-submission inquiries

- Our selector tool helps you to find the most relevant journal

- We provide round the clock customer support

- Convenient online submission

- Thorough peer review

- Inclusion in PubMed and all major indexing services

- Maximum visibility for your research

Submit your manuscript at www.biomedcentral.com/submit
) Biomed Central 